This item was submitted to Loughborough's Research Repository by the author.

Items in Figshare are protected by copyright, with all rights reserved, unless otherwise indicated.

\title{
Morphology of depletant-induced erythrocyte aggregates
}

PLEASE CITE THE PUBLISHED VERSION

https://doi.org/10.1039/c8sm01026a

PUBLISHER

(c) Royal Society of Chemistry

VERSION

AM (Accepted Manuscript)

PUBLISHER STATEMENT

This paper was accepted for publication in the journal Soft Matter and the definitive published version is available at https://doi.org/10.1039/c8sm01026a.

\section{LICENCE}

CC BY-NC-ND 4.0

\section{REPOSITORY RECORD}

Nehring, Austin, Tyler N. Shendruk, and Hendrick W. de Haan. 2019. "Morphology of Depletant-induced Erythrocyte Aggregates". figshare. https://hdl.handle.net/2134/36360. 


\section{Journal Name}

\section{ARTICLE TYPE}

Cite this: DOI: $10.1039 / x x x x x x x x x x$

Received Date

Accepted Date

DOI: $10.1039 / x x x x x x x x x x$

www.rsc.org/journalname

\section{Morphology of depletant-induced erythrocyte aggre- gates}

\author{
Austin Nehring, ${ }^{a}$ Tyler N. Shendruk, ${ }^{b}$ and Hendrick W. de Haan ${ }^{a *}$
}

Red blood cells suspended in quiescent plasma tend to aggregate into multicellular assemblages, including linearly stacked columnar rouleaux, which can reversibly form more complex clusters or branching networks. While these aggregates play an essential role in establishing hemorheological and pathological properties, the biophysics behind their self-assembly into dynamic mesoscopic structures remains under-explored. We employ coarse-grained molecular simulations to model low-hematocrit erythrocytes subject to short-range implicit depletion forces, and demonstrate not only that depletion interactions are sufficient to account for a sudden dispersionaggregate transition, but also that the volume fraction of depletant macromolecules controls small aggregate morphology. We observe a sudden transition from a dispersion to a linear column rouleau, followed by a slow emergence of disorderly amorphous clusters of many short rouleaux at larger volume fractions. This work demonstrates how discocyte topology and short-range, nonspecific, physical interactions are sufficient to self-assemble erythrocytes into various aggregate structures, with markedly different morphologies and biomedical consequences. (a)
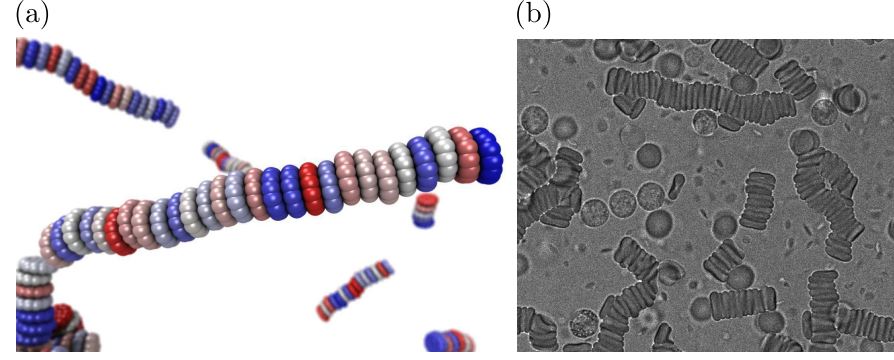

Fig. 1 Images of rouleaux, columnarly ordered aggregates of red blood cells. (a) Simulation snapshot model discocytes. (b) Differential interference contrast (DIC) microscopy image of human erythrocytes in plasma courtesy of Lorena Buitrago (throughout).

\section{Introduction}

Blood is an emblematic biofluid that exhibits complex material behaviour? ? ? ? ? . These physical properties arise not only from blood's soft cellular and biomolecular components, but also the dynamic mesoscopic structures that they form. Within quiescent or weakly sheared plasma, red blood cells (RBC; erythrocytes) tend to aggregate and form multicellular clusters, including striking linear columnar stacks or rouleaux (Fig. ??a) ${ }^{\text {? }}$. These

\footnotetext{
${ }^{a}$ University of Ontario Institute of Technology, Faculty of Science, 2000 Simcoe Street North, Oshawa, Ontario L1H 7K4, Canada

${ }^{b}$ Center for Studies in Physics and Biology, The Rockefeller University, 1230 York Avenue, New York, New York 10065, USA

* Corresponding Author: hendrick.dehaan@uoit.ca
}

mesoscale structures can be reversibly dispersed in increased shear and reform when flow ceases, resulting in complex rheological behavior?

While intense research has focused on microvascular flows? ? and the dynamics of single cells in shear flow? ? ? ? (as reviewed by Secomb? ), the physical origins of structure and the formation of columnar rouleau and other erythrocyte aggregates should not be overlooked. In many pathological cases, an increase in aggregation is reported? ?. Even in simplified microfluidic systems, aggregations of red blood cells can block capillaries prevent-

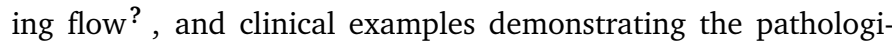
cal importance of aggregates include sickle cell diseases? ?, infections? ? , cardiovascular diseases ? ? and many more ? ? Furthermore, RBC aggregation is correlated with inflammation? , in which acute phase reactants, such as fibrinogen, lead to enhanced aggregation? ?

In many of these cases, aggregation and rouleaux formation follows an increase in concentration of fibrinogen or other high molecular weight proteins in plasma. In fact, RBC aggregation is seen to generically depend on the concentration of high molecular weight macromolecules in vitro? ? an observation that suggests entropic depletion forces? are crucial to the adhesion that leads to mesostructures. Due to their finite size, macromolecules (referred to as depletants in this context) cannot access a thin sheath of excluded volume around each separate RBC (Fig. ??a; top); such that, when two RBCs are in contact, their excluded volumes overlap, increasing the total volume available to the many 
(a)
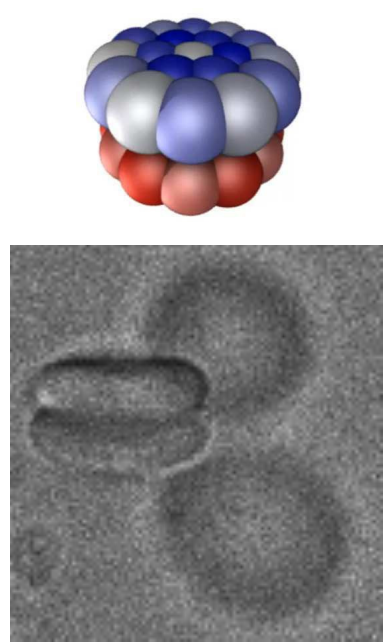

(b)
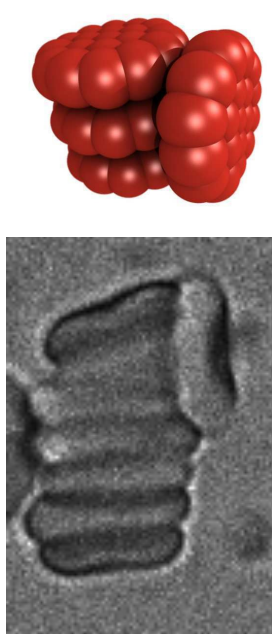

(c)
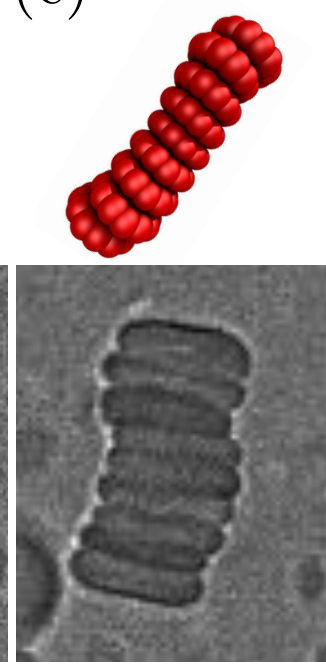

(d)
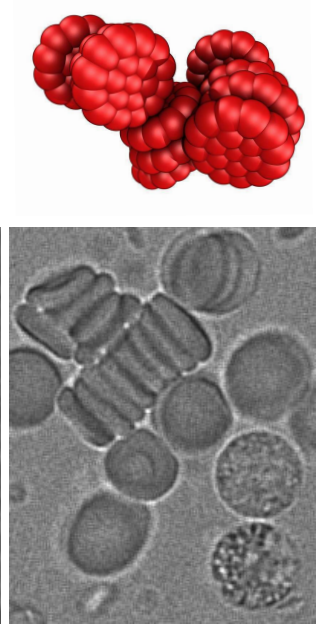

Fig. 2 Typical structures observed in red blood cell aggregates. Above: Simulation snapshots. Below: DIC microscopy images of erythrocytes. (a) A doublet of two RBCs in a "face-to-face" configuration. (b) Three RBCs in a triplet rouleau with a fourth RBC in perpendicular flanking contact. We refer to this state as the "face-to-side" configuration. (c) RBCs aligned into a rouleau configuration. (d) An amorphous cluster of shorter rouleaux.

depletants (Fig. ??a; bottom) and resulting in an entropic attractive force between cells. Depletant-induced entropic attraction is common in biological systems and even thought to be a driver of structural changes in prokaryotic chromosomal material? ? ? In recent years, experimental evidence has concluded that this so-called chemiosmotic hypothesis is necessary (if not necessarily sufficient) for RBC aggregation? ? However, it is important to also recognize that specific macromolecules can interact with the surfaces of two RBCs forming short macromolecular bridges that may tether RBCs? . Some molecules, perhaps including the experimentally important neutral dextran, may indeed act simultaneously as a depletant and bridging macromolecule? ? ? ? .

Despite such intense interest in microvascular rheology and the nature of red blood cell adhesion, the morphology of erythrocyte aggregates has not yet received sufficient scientific consideration. Rouleaux, the long linear columns of neatly stacked RBCs (Fig. ??c), are the most notable state of aggregation. However, short doublets or triplets are commonly observed (Fig. ??a), as are branched rouleaux (Fig. ??b shows a triplet rouleau with a flanking RBC, which can act as a branching point). Additionally, amorphous clusters of many short rouleaux are commonly observed (Fig. ??d). Disorderly clusters, sometimes referred to as hemagglutinates in the medical literature, can signal that antibodies have recognized pathogenic antigens. ? Regardless of the nature of the short-ranged interactions, RBCs essentially behave as soft "sticky" discocytes. While this might lead one to consider diffusion-limited aggregation, it is known from discotic liquid crystals? and solutions of nano-discs? that anisotropic shape allows symmetry breaking and more complicated orientational mesophases, which have been observed in vesicle-based simulations? ?

To study the biophysics of RBC aggregation, we take a simplified approach to modeling discotic erythrocyte and their shortranged interactions. Using a series of coarse-grained simulations that implicitly account for thermal and depletant-induced forces, we demonstrate that non-specific depletants are sufficient to aggregate both columnar rouleau and amorphous clusters. We focus our interest on the transition from dispersed cells to aggregates composed of relatively few RBCs. We see that the low-hematocrit dispersion-aggregate transition is sharp and hysteretic. Additionally, we explore the interaction energy and average lifetimes for different configurations to gain insight into the resulting aggregate morphologies.

\section{Simulations}

Our approach is to perform coarse-grained simulations of model erythrocytes to gain physical understanding of the aggregates. To this end, we ignore erythrocyte biconcavity and deformability as

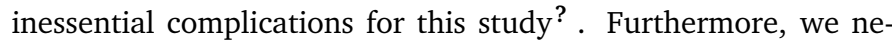
glect the glycocalyx and any specific macromolecular bridging mechanisms. Strictly speaking, we study the chemiosmotic hypothesis that generic depletant-induced forces are the sole cause of attraction; however, we recognize that in clinical systems both effects contribute to erythrocyte aggregation. We posit that depletion forces can act as a convenient and sufficiently generic surrogate for the totality of specific and non-specific "sticky" interactions? .

\section{Model erythrocytes}

Standard coarse-grained molecular dynamics approaches are used to simulate red blood cells? . Each RBC is constructed from molecular dynamics spherical beads. As shown in Fig. ??b, a total of 19 particles are used to build one RBC. One particle is placed at the centre and is surrounded by an inner ring of 6 particles and an outer ring of 12 particles, resulting in a raspberry-type model ${ }^{\text {? }}$. To model excluded volume, each bead has a repulsive interaction with its neighbours and the particles are placed such that they overlap to smooth the surface and thus fill in other- 
(a)

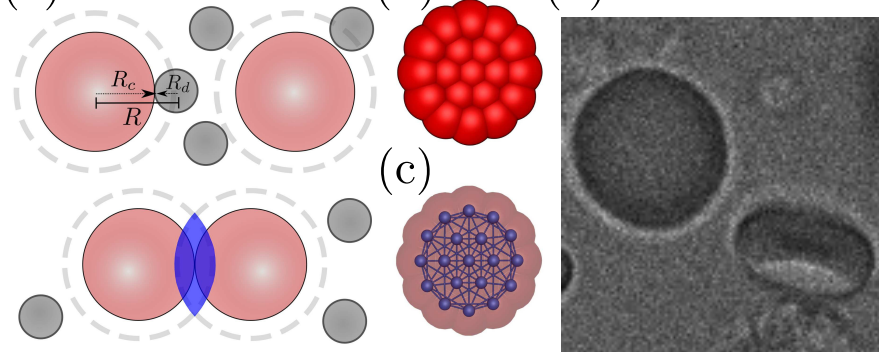

Fig. 3 (a) Diagram illustrating the entropic basis of depletion interactions, in which two colloids of radius $R_{c}$ are suspended in a bath of depletants of size $R_{d}$ (above). When the two colloids make contact, their excluded volumes overlap and the depletants gain free volume, producing a net entropic gain and depletant-induced attraction (below). (b) Structure of the model discocytes, comprised of 19 spheres: 1 central sphere, 6 in the interior ring, and 12 spheres on the exterior ring. (c) Skeleton of the FENE bonds between the 19 spheres represented by blue lines. These bonds, as well harmonic angle potentials and dihedral potentials, are used to maintain the discotic shape. The diameter of the model RBC is $3 \sigma$ and the thickness is $1 \sigma$. (d) DIC microscopy image of two singlet erythrocytes.

wise deep grooves. Pairs of molecular dynamics beads are linked with nonlinear springs and the disk-like shape is sustained by angle and torsion potentials. Similar coarse-grained (or lowdimensional) models of erythrocytes have been successfully applied to study hemorheological flows? ? Though we describe the coarse-grained simulation parameters used here, the details of standard molecular dynamics simulations are reviewed elsewhere? ? ? . The short-ranged radial depletion forces that act between all particles will be discussed in Section ??.

Excluded volume is implemented via the radial WCA potential $^{?}$, with the characteristic energy set to $\varepsilon=1$, nominal diameter $\sigma=1$ and mass $m=1$. Each bead is subjected to thermal fluctuations with a typical thermal energy $k T=1 \varepsilon$. The FENE potential is used to bond particles together and, as in the polymer model of Kremer and Grest ${ }^{\text {? }}$, the spring constant is set to $30 \varepsilon / \sigma^{2}$ with a maximum bond distance $1.5 \sigma$ (Fig. ??c). The FENE potential does not provide the model RBC with rigidity - harmonic angle potentials (with spring constant $45 \varepsilon / \sigma^{2}$ ) are utilized between each pair of antipodal beads on the perimeter and the central bead. This harmonic angle potential prevents the discocytes from folding and constrains the central bead from fluctuating vertically out of the face of the disk. Dihedral potentials (spring constant $45 \varepsilon / \sigma^{2}$ ) are applied to each quartet of spheres in incremental order around the of the rim. This dihedral potential resists torsion of the outer edge and decreases the overall deformability of the model RBC by working in tandem with the harmonic angle potential to maintain the disk-like shape.

These 3 types of bonds ensure that the disk has internal degrees of freedom and possesses flexibility, while also maintaining a disk shape. This construction with its many internal degrees of freedom is redolent of the flexibility that membranous red blood cells exhibit but the parameters are chosen to qualitatively match the behaviour of RBCs but are not intended to quantitatively equate to erythrocyte deformability? ? . The resulting model discocyte has a height of $1 \sigma$ and diameter of $3 \sigma$, the ratio of which is a reasonable approximation to typical erythrocytes with thicknesses of $\approx 2 \mu \mathrm{m}$ and diameters of $\approx 8 \mu \mathrm{m}^{?}$.

Simulations are performed using the HOOMD-Blue simulation package? ? with 100 integration steps per unit time $\tau=\sigma \sqrt{m / \varepsilon}=$ 1. Values throughout are reported in simulation units. Unless otherwise stated, 10 RBCs are included in each simulation of a cubic volume with periodic boundary conditions with a linear system size of $20 \sigma$. By choosing to limit our study to small systems sizes, we are able to focus on the mesoscale structure of small aggregates in the low hematocrit (low RBC volume fraction) limit. In larger quiescent systems, many long rouleaux can form complex networks with many branching points, though these large structures are not typically observed when mild shearing is applied.

The translational diffusion is calculated by measuring the displacement of the center of mass of a single model erythrocyte in an empty volume. The rotational diffusion is determined by tracking the normal vector over time. By measuring the translational and rotational mean squared displacements, we find that the model erythrocytes have translational and rotational diffusion coefficients of $D=0.010 \sigma^{2} \tau^{-1} \pm 0.004 \%$ and $D_{\mathrm{R}}=0.069 \tau^{-1} \pm 0.06 \%$, respectively. The rotational diffusion coefficient provides the characteristic thermal time scale $\tau_{\mathrm{R}} \approx 3 \times 10^{4} \tau$ to traverse $\pi / 2$ radians.

\section{Simulation Procedures}

Three different types of simulations are performed.

Quenched aggregation The first explores the aggregation of erythrocytes into clusters from a dispersed initialization. These are initialized with the RBCs well separated on a grid then simulated for $\sim 70 \tau_{\mathrm{R}}$ in the absence of depletants to randomize the positions. Following this, the depletion interaction is applied and the system is again allowed to equilibrate for $1700 \tau_{R}$. Data is then acquired over $680 \tau_{R}$. This process amounts to a quenched simulation and results are reported in Section ??.

Rouleau dispersion The second type of simulation examines the stability of a pre-existing rouleau. These simulations start with the red blood cells aligned in a linear column rouleau (Fig. ??c). During a warm-up phase, the thermal energy is increased from $k T=0$ to 1 over $34 \tau_{\mathrm{R}}$, after which data is collected for $680 \tau_{\mathrm{R}}$ at constant temperature. These simulations are presented in Section ??.

Interaction stability The final type of simulations provides data on the interaction energy between specific contact configurations and their lifetimes. In these simulations, we consider a doublet, initialized in face-to-face contact (Fig. ??a), or a triplet rouleau and flanking fourth RBC with its face on the side of the column (Fig. ??b). Data is acquired over $680 \tau_{\mathrm{R}}$. Simulation from these starting conditions illustrate the stability and lifetime of aggregates, and are discussed in Section ??

\section{Aggregate analysis}

To characterize the aggregates in simulations, three different measurements are used: The averaged number of clusters, max- 
imum size cluster size, and number of aligned RBCs. All three quantities are calculated by generating an adjacency graph for the cells in the simulation. Once the adjacency graph is created the number of connected components is calculated; this directly retrieves each quantity. Calculations of the adjacency graphs are accomplished using the NetworkX python package. ?

The criteria for two RBCs being in a cluster is based on the sphere to sphere distance of the particles. If any two particles in the RBCs are less than $(\sigma+0.05 \sigma)$ then the cells are considered in contact. The conditions for being aligned are more strict: Not only does the cell need to be in the cluster, but the center-tocenter distance of each RBC must also be less than $\sqrt{2} \sigma$. These measurements allows us to discriminate between states.

Dispersions Little aggregation observed when attraction does not contend with thermal agitation.

Columnar rouleaux When all erythrocytes in an aggregate are linearly aligned they form a rouleau (Fig. ??c).

Amorphous clusters Many short rouleaux can aggregate into clusters, which may or may not be aligned (Fig. ??d).

\section{Depletion-induced attractions}

To investigate depletion-induced aggregation, simulations must be performed across many volume fractions and explicitly including depletants would be computationally intensive. Instead we utilize the implicit and analytical Asakura-Oosawa (AO) pair potential between spheres? ? ? To understand the cause of depletion forces consider two spheres in a bath of many smaller depletant particles (commonly proteins or macromolecules in plasma). The macromolecular depletants cannot fit within a thin layer around each of the separate spheres because of their finite size (Fig. ??a; above). However, when the large spheres are close together these two exclusion layers overlap (Fig. ??a; below), causing the overall volume accessible to the depletants to increase slightly. The increased volume is accompanied by an increase in the depletants' entropy, which enacts an osmotic pressure or short-ranged effective attractive force that pushes the two spheres together. While the two large spheres in Fig. ??a do lose some entropy when held together, the ensemble of depletants' entropy gain is much greater because there are so many more of them.

In the dilute limit of depletants, the osmotic pressure is approximated by the van't Hoff equation $\Pi=-\phi k T / V_{d}$, for spherical depletants of volume $V_{d}$ and radius $R_{d}$. The osmotic pressure times the overlap volume $V(r)$ produces an effective pair potential $U(r)=\Pi \cdot V(r)$. Increasing the volume fraction $\phi$ deepens the entropic attraction. For spherical colloids of radius $R_{c}$, the AO pair potential? ${ }^{\text {is }}$

$$
\frac{U_{\mathrm{AO}}}{k T}=-\left(\frac{R}{R_{\mathrm{d}}}\right)^{3}\left[1-\frac{3}{4} \frac{r}{R}+\frac{1}{16}\left(\frac{r}{R}\right)^{3}\right] \phi .
$$

where $R=R_{c}+R_{d}$ (Fig. ??a) and $r$ is the center-to-center separation. The AO potential is limited by a hard-sphere potential when $r<2 R_{c}$ and is only non-zero while $r<2 R$. The potential's depth is $U_{\mathrm{AO}}^{\min }=-\left(1+\frac{3}{2} \frac{R_{c}}{R_{d}}\right) \phi k T$. In our simulations, $R_{c}$ corresponds to $0.5 \sigma$ and we use Eq. ?? between all spheres in the simulations, setting $R_{c} / R_{d}=25$. This ratio is smaller than measured for plasma proteins and other RBC relevant macromolecules but is the correct order of magnitude? .

The AO potential used in the simulations describes the interaction between spherical particles. Additionally, we calculate the interaction energy between rouleaux modelled as two cylinders with effective radii $R=R_{\mathrm{RBC}}+R_{d}$ and effective lengths $L_{1}$ and $L_{2}$ (cylinder height plus $2 R_{d}$ ) in a variety of configurations. The potentials are in terms of the separation between the cylinders' centres $(r, z)$. For brevity, note that the overlap area between two circles is $2 R^{2} \cdot A(r / 2 R)$, where $A(x)=\cos ^{-1}(x)-x \sqrt{(1-x)(1+x)}$.

Face-to-face columnar stacking (Fig. ??a) The face-to-face interaction potential of two cylindrical RBCs is

$$
\frac{U_{\mathrm{ff}}}{k T}=-\frac{3}{4 \pi}\left(\frac{R}{R_{d}}\right)^{2}\left(\frac{L_{1}+L_{2}-2|z|}{R_{d}}\right) A\left(\frac{r}{2 R}\right) \phi .
$$

The well-depth is $U_{\mathrm{ff}}^{\min }=-(3 / 2)\left(R / R_{d}\right)^{2} \phi k T$, which is $-8664 \phi k T$ for the simulation parameters. This value should be compared to $U_{\mathrm{AO}}^{\min }=-113.5 \phi k T$, the depletion energy of two spheres of radius $R_{\mathrm{RBC}}$ in contact. The fact that $U_{\mathrm{ff}}^{\min } \gg U_{\mathrm{AO}}^{\min }$ is indicative of the importance of shape in the strength of depletant-induced attractions.

Face-to-side contact (Fig. ??b) When the cylindrical side of rouleau 1 approaches the face of rouleau 2, a face-to-side perpendicular flanking contact occurs. For cases in which the face of cylinder 2 is not in contact with the ends of cylinder 1 , the overlap volume normalized by $R^{3}$ is

$$
\mathscr{V}_{\circ}=\left(2-h^{2}\right) \sqrt{1-h^{2}}-h \cos ^{-1}(h)
$$

where $h(r)=1-H / R$ and $H(r)=R+L_{2} / 2-r$ is the radial overlap. At contact $H=2 R_{d}$. This accounts for both the curvature of cylinder 1 and the circular area of cylinder 2 . However, if the finite length of cylinder 1 matters, we neglect the circularity of the face and over-approximate the normalized volume to be $\mathscr{V}_{\text {lin }}=$ $\lambda(z / R) A(h)$, where $\lambda(z / R)=\min \left[2, L_{1} / R, 1+\left(L_{1}-2 R_{d}-z\right) / R\right]$ is the normalized axial overlap height. Choosing the most accurate form of the overlap volume approximates the depletion pairpotential as

$$
\frac{U_{\mathrm{fs}}}{k T}=-\frac{R^{3}}{V_{d}} \min \left[\mathscr{V}_{\circ}\left(\frac{r}{R}\right), \mathscr{V}_{\operatorname{lin}}\left(\frac{z}{R}, \frac{r}{R}\right)\right] \phi
$$

For $L_{1}>2 R$ and small depletants, $\mathscr{V}_{\circ} \approx 32\left(R_{d} / R\right)^{3 / 2} / 3$ and $U_{\mathrm{fs}}^{\min } \approx$ $(8 / \pi)\left(R / R_{d}\right)^{3 / 2} \phi k T$. In this case, the minimum is $U_{\mathrm{fs}}^{\min }=$ $-1687.2 \phi k T$. While this is less than the face-to-face interaction energy, it is also an order of magnitude greater than $U_{\mathrm{AO}}^{\min }$ for two spheres of radii $R_{\mathrm{RBC}}$.

While face-to-face columnar stacking is most energetically favourable, it is clear from these considerations that the interaction energy of face-to-side contacts can be of the same order of magnitude. This may introduce biaxial orientational ordering in aggregates. 


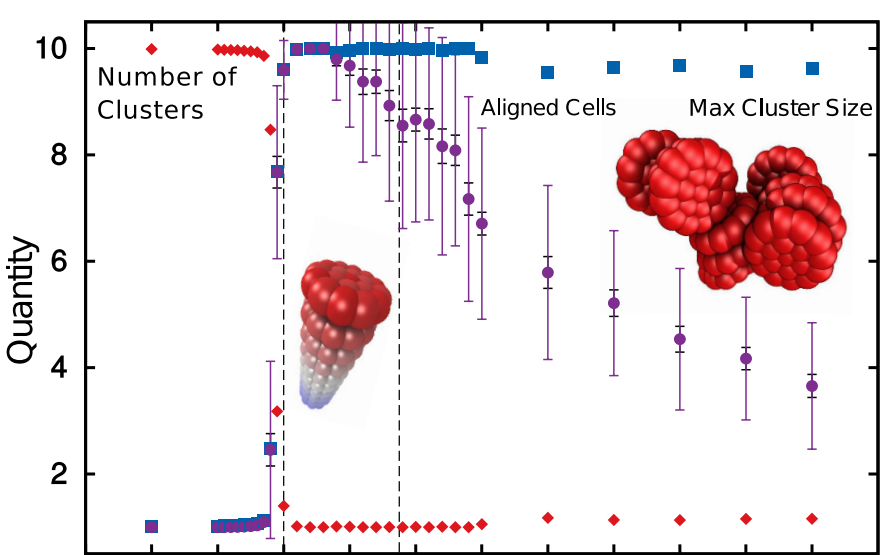

0.040 .050 .060 .070 .080 .090 .10 .110 .120 .130 .140 .15 Volume fraction $-\phi$

Fig. 4 Quantification of quenched aggregation of red blood cells. At low depletant volume fractions, the RBCs are dispersed as single cells and the average number of clusters is (red diamonds) equals the number of RBCs in the system, while the average maximum cluster size (blue circles) is 1 . At intermediate volume fractions, a transition occurs, indicating a sudden onset of aggregation. The morphology of the aggregate is indicated by the time averaged maximum number of aligned RBCs (purple), which is seen to have a maximum near the transition but decays at larger volume fractions indicating a greater likelihood of forming amorphous clusters. Larger error bars show the standard deviation of the distribution, while smaller error bars give the uncertainty on the mean (throughout). Dashed lines indicate $\phi_{\mathrm{ff}}^{*}$ and $\phi_{\mathrm{fs}}^{*}$, the respective volume fractions at which face-to-face and face-to-side configurations are long lived.

\section{Lattice-based canonical ensemble}

Having calculated the depletion interaction energies, aggregate attributes can be estimated by constructing an approximate lattice-based model of the canonical ensemble. In this model rouleaux are treated as smooth hard cylinders, as in Section ??. By discretizing the system's volume and neglecting all but the most energetically significant cluster configurations, we will enumerate each microstate to approximate the canonical partition function $Z$. Doing so estimates the probability of each microstate, which in turn allows this lattice model to approximate the thermodynamic ensemble averages. Explicitly enumerating the microstates is tractable because we consider small systems of $N=10$ RBCs. While our computational results will demonstrate that amorphous hemagglutinates are frozen in deep metastable states (Sections ??), we will understand these morphologies relative to ensemble averages in thermal equilibrium.

The discrete model is constructed by asking: a. In how many ways can RBCs be grouped into rouleaux? b. In how many ways can those rouleaux interact to form clusters? c. What is the energy and probability of each microstate?

a. There are multiple ways that $N$ RBCs can be partitioned into various groupings (with each group representing a single linear rouleau). In our case, the integer partition function is $p(10)=$ 42 distinct ways of forming rouleaux from $N=10$ RBCs. This includes a single rouleau of $n=10 \mathrm{RBC}$, ten single cell "rouleaux" ( $n=1)$, two rouleaux of $n=5 \mathrm{RBCs}$ each, and every partition between. In this model, we assume that each pair of RBCs in a rouleau interacts with energy $U_{\mathrm{ff}}^{\min }$ from Eq. ??, such that the approximate interaction energy of a single rouleau of $n$ RBCs is simplu $(n-1) U_{\mathrm{ff}}^{\mathrm{min}}$.

b. For each of the $p(N)$ partitions, the resulting rouleaux can be dispersed in solution or cluster into aggregates. Figures ??b and ??d illustrate that aggregates can have potentially complex structures; however, we make the simplifying assumption that clusters have a central rouleau and up to four rouleaux can adhere to it $(m \leq 1+4)$. This assumption limits the size of clusters that we consider here and assumes that $m>5$ clusters are unlikely, which simulations show is reasonable for $N=10$ (Fig. ??d) but may not be true for larger systems since we neglect chains of many rouleaux (Fig. ??; inset) and central rouleaux that are long enough to accommodate more than four adherents. Algorithm $U$ is employed to find all sets of aggregates made of $\leq m$ rouleaux? . Since the interactions between pairs of rouleaux can depend on rouleau length (see Eq. ?? or the Appendix), all distinct configurations of the $m$ rouleaux within each cluster are enumerated. Following Flory lattice theory of polymer solutions, the clusters are placed on a cubic lattice with small lattice site volume (we use $2 \cdot 10^{18}$ lattice sites). The number of translational states are given by successively placing each cluster and taking into account excluded volume by deducting the occupied lattice sites from consecutive placements.

c. Within each cluster, each of the $m-1$ rouleau has a depletioninduced pair-interaction with the central rouleau. Various configurations and associated interaction potentials are possible. Faceto-side contact, with an interaction energy of $U_{\mathrm{fs}}^{\min }$ was found from Eq. ?? but other configurations are possible, including crossing configurations and parallel alignment. As detailed in the Appendix, crossing configurations are least energetically favourable and parallel alignment is only likely when both rouleaux are long. Together, these energies suggest that parallel and perpendicular contacts between rouleaux are energetically negligible compared to face-to-face and face-to-side conformations. Therefore, although all possible interactions should be permuted, we neglect all rouleau-rouleau interactions, except face-to-side contacts (Appendix).

The energy $E_{i}$ of each microstate $i$ is then known to be the interaction energy between each RBC in each rouleau and the interaction energy between rouleaux in each cluster. The canonical partition function $Z=\sum_{i} e^{-E_{i} / k T}$ can be explicitly calculated for this lattice-model of smooth, cylindrical rouleaux and the probability of each state $p_{i}$ can be calculated, allowing ensemble averages, such as the average number of clusters and the average number of aligned, to be predicted. However, because the theory is for smooth hard cylinders while the simulations are of model discocytes composed of 19 soft beads (Fig. ??b-c), we scale $U_{\mathrm{ff}}^{\min }$ by $19 c$, where $c$ is near unity, to compare directly to simulations. As we will discuss in detail in Section ??, face-to-face contact is stable on the time scale of the simulations at $\phi=0.07$ where the total interaction energy is 37.8. Matching this simulation energy to $U_{\mathrm{ff}}^{\min } / 19$ sets $c=0.844$. 


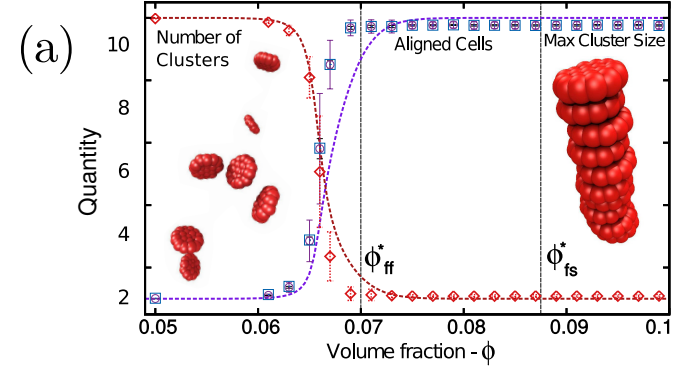

(b)

(c)

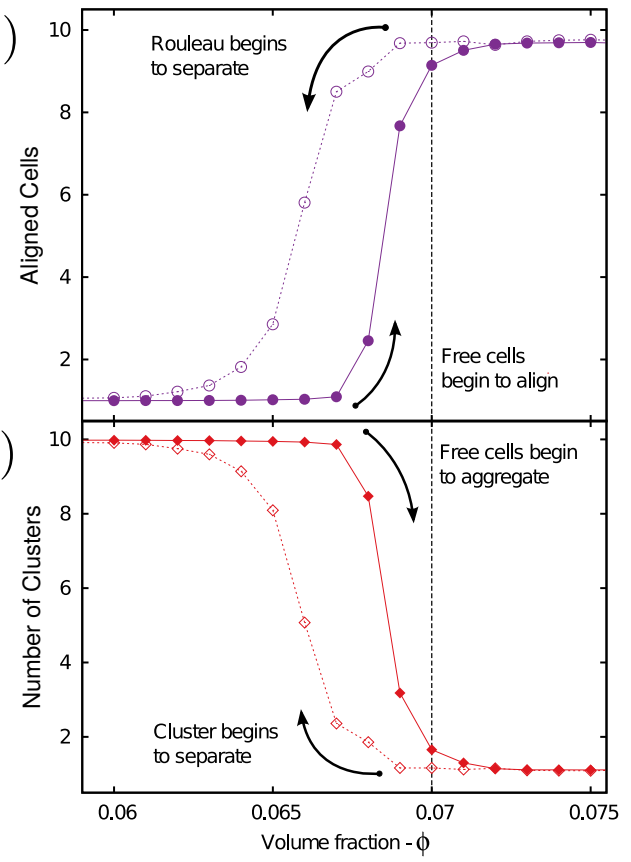

Fig. 5 (a) Same as Fig. ?? but for systems initialized as a single columnar rouleau. The distributions are narrower, the dispersion-aggregate transition is shifted to lower volume fractions and the alignment is not reduced at high volume fractions. (b) Time averaged maximum number of aligned RBCs near the aggregation point for quenched aggregation (filled) and rouleau dispersion (open). The quenched aggregation path decays at greater volume fractions than shown here. The coercivity is approximately 0.003 . The dashed vertical line demarcates $\phi_{\mathrm{ff}}^{*}=0.07$, the volume fraction for which face-to-face contacts are long lived. (c) The same as panel (b) but for the number of clusters. The two curves mirror each other in the vicinity of the dispersion-aggregate transition point.

\section{Results and Discussion}

Using these coarse-grained simulations, depletion pair potentials and the equilibrium approximation, we explore the morphologies of erythrocyte aggregates.

\section{Quenched aggregation}

The averages of the number of clusters, maximum cluster size and number of aligned RBCs are plotted as a function of depletant volume fraction in Fig. ??. At low volume fractions, the depletion forces are insufficient to lead to aggregation. The discocytes are dispersed, each "cluster" is a single cell and so the number of clusters starts is equal to the number of RBCs in the simulation. Correspondingly, both the maximal cluster size and number of aligned RBCs are unity. This behavior continues as the concentration of depletants is increased until $\phi \lesssim 0.07$, at which point the number of clusters suddenly drops to unity, while the average maximal cluster size increases to 10 . This indicates that all the RBCs have suddenly aggregated into a single cluster. The suddenness of this dispersion-aggregate transition is reminiscent of a critical flocculation concentration in colloid science? ?

However, unlike a traditional aggregating particles? ? , the erythrocytes assemble into a highly order columnar structure immediately above critical flocculation concentration of depletants (Fig. ??). Cylindrical structures qualitatively similar to rouleaux arise in columnar phase liquid crystals? . In these systems, disklike particles (such as colloidal nano-discs? ? , clay platelets? disk-shaped-micelles? ? and molecular discogens? ? ?) can stack into columnar structures, which act as mesogens and themselves order. In these soft condensed matter systems, self-assembly of columnar structures leads to liquid crystaline phases with longrange order that can reach macroscopic scales and can be computationally simulated? ? . On the other hand in our present study of model erythrocytes, we focus on the initial, small clusters and their mesoscale structure in the low hematocrit limit.

As $\phi$ increases further, the RBCs remain in a single aggregate since the depletion forces are further strengthened. However, the morphology of the cluster changes. Just after the dispersionaggregate transition, all of the RBCs are aligned with barely any variation. This indicates that the RBCs are aligned in a single columnar rouleau (Fig. ??; leftmost inset). However, around $\phi \approx 0.076$ the mean begins to slowly decrease. Additionally, the distribution of alignments widens substantially at any given $\phi$.

By $\phi=0.09$, a single columnar rouleau is a relatively rare configuration (the maximum is barely within the standard deviation). It is clear that the RBCs no longer align in a single columnar rouleau. Instead, there is continual decay in the maximum number of aligned cells until at $\phi=0.15$ the average maximum is less than 4 aligned cells. At these large volume fractions, the depletion forces are very strong and thus RBCs tend to adhere in whatever configuration they first make contact. Although columnar alignment is still the lowest energy configuration, the depletion forces are strong enough to yield long lasting out-of-equilibrium, or metastable, states. The result is an amorphous morphology reflecting a disorderly cluster of short rouleaux (Fig. ??; rightmost inset).

\section{Rouleau dispersion}

Since the quenched aggregate simulations demonstrate that the simulated hemagglutinates most frequently find deep metastable states, we run simulations initialized in the columnar rouleau state (Fig. ??a). Because the face-to-face attraction is predicted to be so much larger than other configurations (Eq. ??), the columnar rouleau state corresponds to the global energy minimum. Consequently, at high volume fractions the RBCs remain aligned in a single columnar rouleau until at a sufficiently low volume fraction the model discocytes suddenly separate into a dispersed phase in which they do not cluster for any significant period of time.

The distributions are seen to be much narrower than in the quenched simulations since it is unlikely that the system escapes 
from the global energy minimum of the columnar rouleau configuration above the dispersion-aggregate transition. Since the initial columnar rouleau configuration corresponds to the system's global energy minimum, the dispersion-aggregate transition observed in these simulations closely conforms to the equilibrium predictions described in Section ??. Excellent agreement is observed (Fig. ??a), though the theory predicts that rouleaux dispersion occurs at slightly larger $\phi$ than is observed in the simulations. This may be because the statistical mechanics model limits interactions to a number of simple well-defined energy states, whereas the simulations allow internal RBC fluctuations within rouleaux.

Comparing Fig. ?? to Fig. ??a, we see that the dispersionaggregate transition occurs at a slightly lower volume fraction, suggesting hysteresis. To better compare between the systems in the vicinity of the dispersion-aggregate transition, the number of aligned RBCs and number of clusters for both cases are in shown in Fig. ??b,c. It is clear that there is hysteresis for $\phi$ between 0.06 and 0.075 with a finite coercivity of about 0.003 . Hysteresis is expected in the dispersion-aggregation transition, since the process is highly history-dependent, with great number of metastable configurations frozen into nonequilibrium states. Simulations with longer equilibration times are naturally expected to reduce the measured hysteresis; however, we will soon demonstrate that the lifetimes of contacts grow exponentially near the transition.

\section{Contact stability}

Previous studies have explored thermodynamic phase transitions into liquid crystal phases of discotic particles? ?? and blood flow has been computationally studied from this perspective? . However, rather than approaching thermodynamically large numbers of RBCs, we probe contact interactions between basal configurations of rouleaux. Before a rouleau can form, a doublet of two initial RBCs must be stable for a sufficient time that additional cells have the opportunity to interact with it, and likewise, amorphous hemagglutinate clustering requires that that secondary contacts are stable. This motivates us to consider the stability of various simplified configurations. We hypothesize that amorphous clusters occur when the well-depth of both face-toface (Fig. ??a) and face-to-side (Fig. ??b) configuration dominate over thermal energy, while an aligned columnar rouleau is only likely to form in the range of of volume fractions for which the face-to-face doublet is stable but the face-to-side quartet is not.

We perform two types of simulations to test these hypotheses as described in Section ??. First, two RBCs are placed in a face-toface doublet configuration (Fig. ??; inset). Second, a red blood cell is placed with its face against the side of an aligned triplet rouleau (Fig. ??b; inset). In both cases, the time required for the RBCs to separate is recorded, as well as the interaction energies. In the second case, we calculate the interaction energy and lifetime of the cell in the face-to-side configuration. An RBC is considered to have escaped when the distance between every pair of spheres is greater than $(\sigma+0.05 \sigma)$ and the simulations are repeated 100 times.

At low $\phi$, the depletion attraction is weak and the RBCs separate with a time on the order of the timescale to freely diffuse a

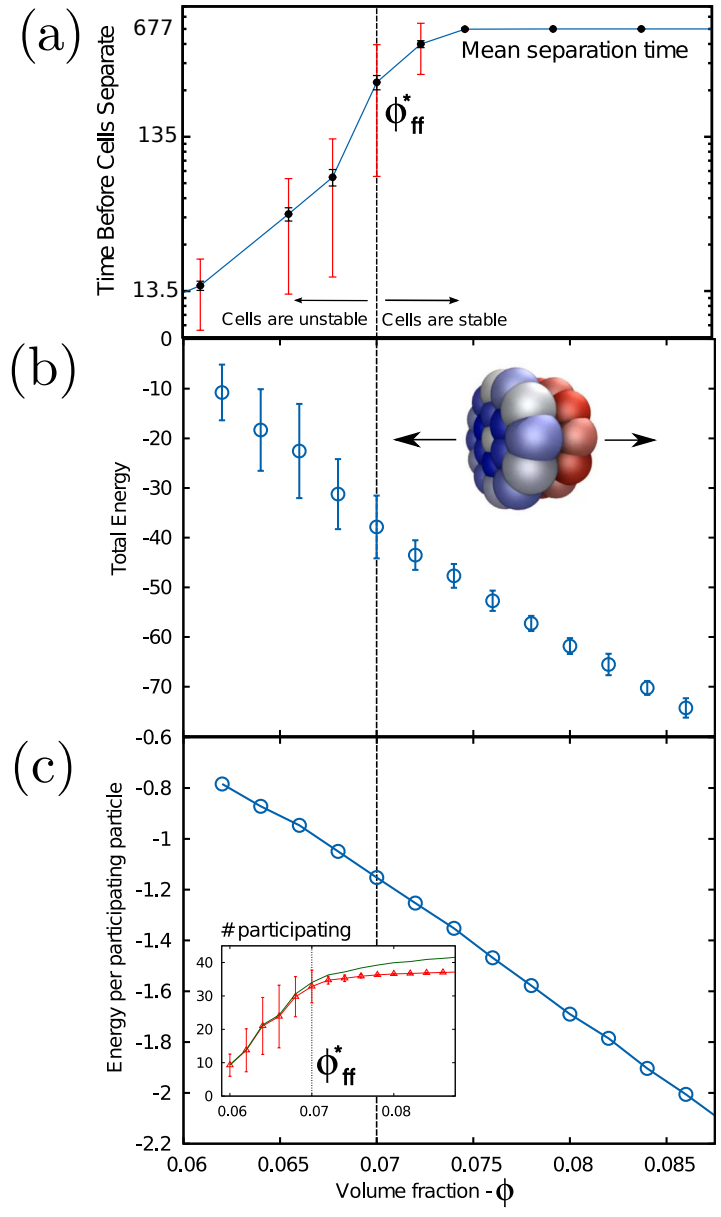

Fig. 6 The stability of face-to-face doublets. (a) The average lifetime of a doublet. At lower volume fractions than shown, the lifetime is constant. Near the dispersion-aggregate transition, the lifetime grows exponentially until $\phi_{\mathrm{ff}}^{*}=0.07$ (vertical dashed line) at which point doublets are likely to survive for more than half the simulation time. We identify this point as the critical volume fraction beyond which face-to-face contacts are stable on the timescale of the simulations. (b) The total interaction energy in simulation units of the face-to-face configuration. (c) The interaction energy normalized by the number of participating particles. (inset) The mean number of particles participating in the interaction (red triangles) rises with volume fraction. Particles can have multiple pair interactions so the total number pair interactions (green line) differs at strong adhesion.

distance of $(\sigma+0.1 \sigma)$. This dilute limit is not shown in Fig. ??a. As $\phi$ approaches the dispersion-aggregate transition, the separation time increases exponentially. The maximum simulation time is 5000 and the data saturates at high $\phi$, meaning that the RBCs are unlikely to separate in the simulated time. The lifetime reaches the midpoint of the total simulation time around $\phi=0.07$ in these doublet simulations. Volume fractions lower than 0.07 correspond to doublets that tend to be unstable, while volume fractions higher tend to be stable. In the vicinity of the midpoint, the variance of the lifetimes is large, indicating that some RBCs separate immediately while others remain adhered for nearly the whole simulation. This again indicates that $\phi=0.07$ lies at the transition between dispersed erythrocytes and columnar rouleau formation. Based on this lifetime evidence, we define the critical volume fraction for which face-to-face configurations are stable 
on macroscopic timescales to be $\phi_{\mathrm{ff}}^{*}=0.07$ (dashed vertical line; Fig. ??-??).

The critical volume fraction $\phi_{\mathrm{ff}}^{*}$ for stable face-to-face doublets and the associated dispersion-aggregate transition corresponds reasonably well to physiological systems. For example, a constant volume fraction of $7.8 \%$ has been utilized to experimentally study the impact of macromolecular size on in vitro RBC aggregation? . Likewise, $\phi_{\mathrm{ff}}^{*}$ is comparable to the depletant volume fraction in whole blood: Plasma constitutes $\sim 55 \%$ by volume of whole blood and about $\sim 90 \%$ of plasma is water. The remainder is principally suspended biomolecules, a significant portion of which is the small globular protein serum albumin. However, these proteins are likely too small to induce aggregation in erythrocytes? . Fibrinogen (at $\sim 5 \%$ of the dry weight of plasma, with molecular weight is $340 \mathrm{kDa}$, and a hydrodynamic radius of $\sim 11 \mathrm{~nm}$ ) and globulins (with a wide range of molecular sizes) are the most likely principle depletant molecules in blood plasma. By approximating $\alpha$-2-macroglobulin (hydrodynamic radius $\sim 9 \mathrm{~nm}$ and molecular weight $725 \mathrm{kDa}^{?}$ ) as a typical globlin, we estimate that plasma depletants have an volume fraction of $\sim 7.5 \%$, in good agreement with $\phi_{\mathrm{ff}}^{*}$.

The total interaction energy is also recorded in the simulations (Fig. ??b). As we expect for depletion forces in the van't Hoff limit, the interaction energy linearly follows the depletant volume fraction. At $\phi_{\mathrm{ff}}^{*}$, the energy is $-37.8 k T$, which indicates adhesion that it is many times the thermal energy; however, the model discocytes are not rigid disks, but rather are composed of many individual particles joined together and so have many internal degrees of freedom. To account for this, the total number of particles that are participating in the depletion interactions is calculated (Fig. ??c; inset). The average number of particles participating in the doublet contact increases with $\phi$. Again, there is a sudden decrease in the variance, indicating a stable value. Beyond $\phi_{\mathrm{ff}}^{*}$ the number of participating particles saturates to 38 indicating that every particle in both discocytes is in contact. The energy normalized by the number of participating particles is around $-1.1 k T$ at $\phi_{\mathrm{ff}}^{*}$. This energy agrees with the intuition that aggregation occurs when the depletion interactions are stronger than the thermal energy.

This series of calculations is repeated for the face-to-side configurations (Fig. ??). The results are qualitatively similar to the face-to-face doublet, but the critical volume fraction is now at a larger value and we define $\phi_{\mathrm{fs}}^{*}=0.0875$ to be the point at which the cluster takes an average of half the total simulation to separate. The transition region with large variance is now broader, indicating that the behavior in the face-to-side case is more varied than the face-to-face case.

The total depletion energy is around $-30 k T$ at $\phi_{\mathrm{fs}}^{*}$ (Fig. ??b), which is similar to the face-to-face case. However, the energy has larger variance. When RBCs are sticking together in a face-to-face configuration, the discocytes are quite immobile with respect to one another, while a red blood cell that is adhered to the side of a rouleau is free to explore all the cylindrically equivalent conformational states on the surface of the triplet rouleau. Likewise, the number of participating particles saturates around 20 indicating that the rouleau's curvature does not allow every particle

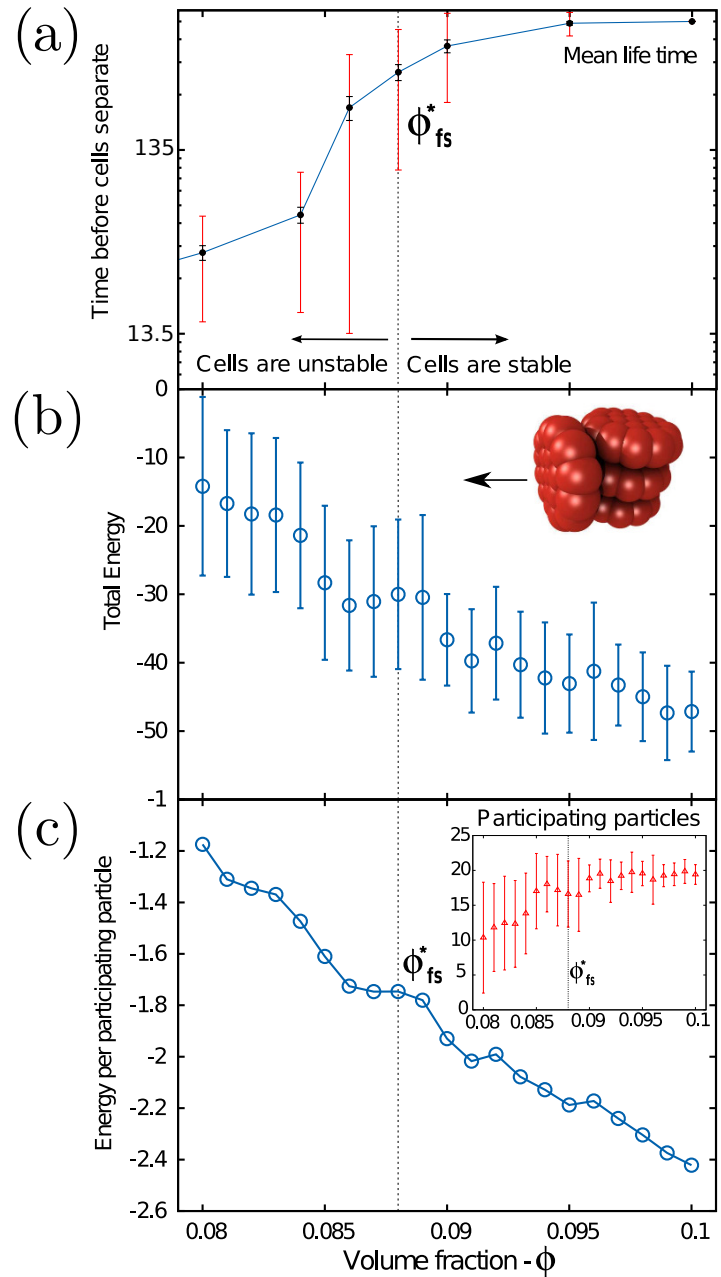

Fig. 7 Same as Fig. ?? but for the face-to-side configuration. The dashed vertical line now marks the $\phi_{\mathrm{fs}}^{*}=0.0875$, above which it is probable that face-to-side aggregates endure for more than half the simulation time.

to interact in this configuration. This is reflected in the analytical result that $U_{\mathrm{fs}}^{\min }<U_{\mathrm{ff}}^{\min }$. The normalized energy (Fig. ??c) at $\phi_{\mathrm{fs}}^{*}$ is around $-1.7 k T$, which is deeper than the $\phi_{\mathrm{ff}}^{*}$ value. Since fewer particles are in contact, internal discocyte fluctuations are enhanced and the variance in the energy is greater than the faceto-face case. This expedites separation and thus the average adhsion per particle must be greater for $\phi_{\mathrm{fs}}^{*}$ than $\phi_{\mathrm{ff}}^{*}$.

\section{Hematocrit effects}

The results so far have been restricted to a single hematocrit or red blood cell number density. We next consider the impact of finite RBC number and hematocrit on rouleau and cluster formation in the dilute limit. Two additional systems are simulated to explore this issue. In the first, the volume of the simulation box is kept constant but the number of RBCs is doubled from 10 to 20 . Hence, these simulations are performed at a higher RBC density. In the second, the discocyte density is maintained by again doubling the number of RBCs but also appropriately increasing the system volume.

Comparing between the average normalized number of clusters, only slight differences in the dispersion-aggregate transition 
region are apparent (Fig. ??a). In fact, results at constant hematocrit (Fig. ??a; blue and green) are quite similar, demonstrating only minor finite number effects. However when the number density is doubled, the steepness of the transition increases. Nonetheless, $\phi_{\mathrm{ff}}^{*}$ continues to accurately describe the point for which total aggregation has occurred. Future research should further consider systems of increased RBC number and hematocrit to identify the possible existence of a critical point.

While we have shown that the dilute limit dispersion-aggregate transition is only weakly impacted by hematocrit, this is not the case for cluster morphology (Fig. ??b). For each system, the average number of aligned RBCs rises suddenly to nearly the total number of model erythrocytes just after $\phi_{\mathrm{ff}}^{*}$. We recall that these aggregates are most often a single columnar rouleau (see Section ??). The peak of the average number of aligned RBCs is seen to be broader for the 10 cell case than it it is for either of the 20 simulations, regardless of RBC number density. The range above $\phi_{\mathrm{ff}}^{*}$ for which a single columnar rouleau is likely to be observed decreases as the number of RBCs in the system increases. For large numbers of erythrocytes, perfect rouleau may only be likely immediately above the dispersion-aggregate transition. Indeed, we qualitatively observe in simulations with a larger number of RBCs that the formation of two long columnar rouleaux often form, before coming in contact and sticking in a non-orientationally aligned conformation. This process is akin to diffusion-limited cluster aggregation in colloidal science? but with the complication that both individual erythrocytes and aggregated rouleaux are anisotropic structures. The result of this anisotropy can be observed in the steady decrease in the average number of aligned RBCs beyond $\phi_{\mathrm{fs}}^{*}$ (Fig. ??b).

At large depletant volume fractions the adhesion dominates over thermal fluctuations and these states are long lived, yielding amorphous clusters of short rouleaux (Fig. ??; inset). Interestingly, the number of aligned cells nearly converge for large $\phi$ to about 4-5 aligned RBCs. We can qualitatively understand this limit by considering the probability that a dispersed RBC first makes contact in the face-to-side configuration or face-to-face. As in the theoretical depletion potentials, we approximate the rouleau as an ideal cylinder of height $L$. Any point of the incoming discocyte can make face-to-side contact over the surface area of $a_{\mathrm{fs}}=2 \pi R_{\mathrm{RBC}}\left(L+R_{\mathrm{RBC}}\right)$. On the other hand face-to-face contact (at either the top or bottom) occurs over the surface area $a_{\mathrm{ff}}=2 \cdot \pi\left(2 R_{\mathrm{RBC}}\right)^{2}$. Thus, we expect incoming face-to-side contact to be more likely when $L \gtrsim 3 R_{\mathrm{RBC}}$, corresponding to rouleau composed of $4-5 \mathrm{RBCs}$ in good agreement with the high $\phi$ limit of amorphous clusters (Fig. ??b). This is qualitatively confirmed in simulation snapshots of amorphous clusters in which quartet and quintet columnar rouleaux are observed but longer stacks are not.

\section{Conclusion}

The morphology of red blood cell aggregates is highly non-trivial; however, we have shown that much of the structure can be understood generically as a biophysical phenomenon arising from topological and energetic considerations. We studied the formation of aggregates due to a well-defined short-ranged depletion attraction using numerical simulations. While complicated deple- (a)
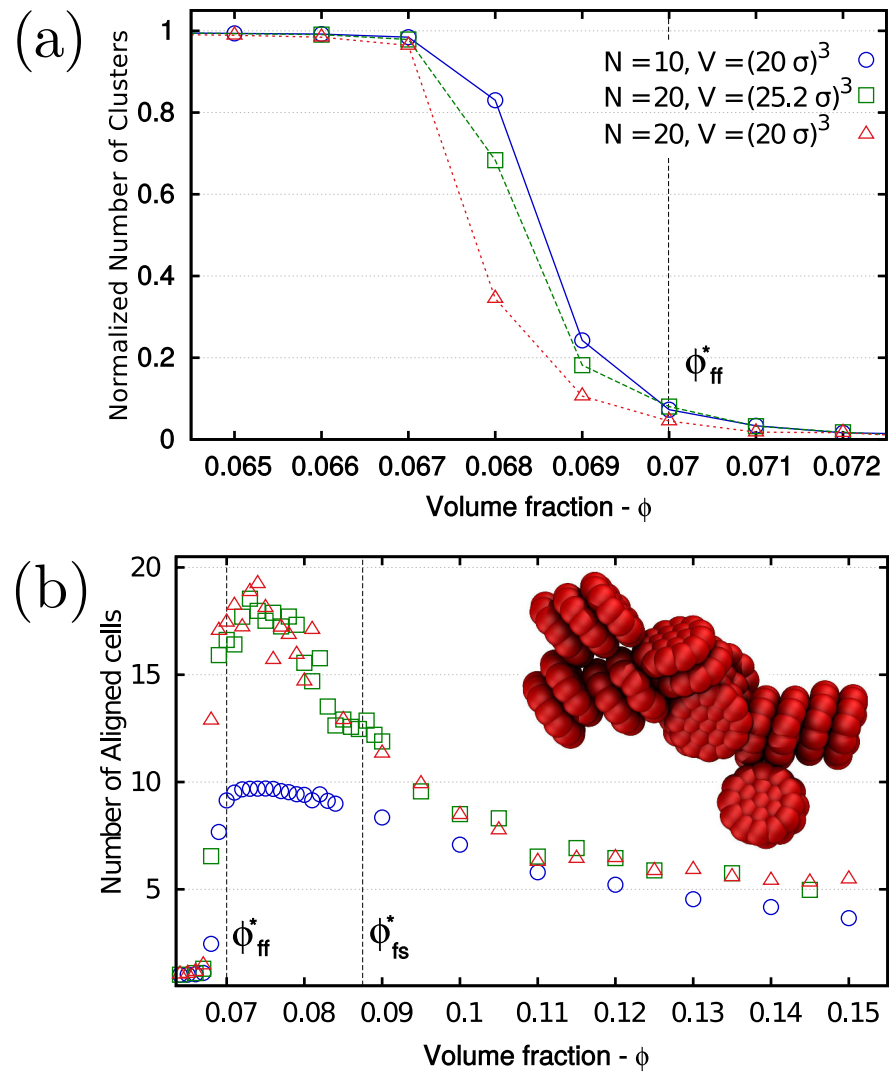

Fig. 8 Comparison of hematocrits effects for systems of 10 and 20 red blood cells. (a) The number of clusters $n$ normalized by the number of RBC, $(n-1) /(N-1)$ for a system of 10 RBCs (blue), a system of 20 RBCs with the same number density (green) and a system of 20 RBCs with higher density (red) in the vicinity of the dispersion-aggregate transition. (b) The average maximal number of aligned RBCs for the three systems. (inset) A simulation snapshot of $20 \mathrm{RBCs}$ in an amorphous cluster of short rouleaux.

tion pair potentials or biochemically specific bridging mechanisms were not accounted for, our simulations reproduced much of the rich variety of clinically observed states, including dispersed cells, rouleaux and amorphous hemagglutinates. The morphology of the aggregates depends strongly on the volume fraction of implicitly depletants. The self-assembly of long, orderly and aligned columnar rouleaux was only observed for a narrow range of depletant volume fractions.

In addition to demonstrating that generic entropic interactions at reasonable volume fractions are sufficient to account for both rouleaux and even hemagglutinate formation, our observations reflect the fact that rouleau formation is fundamentally a consequence of the anisotropy of erythrocyte cells. Understanding how geometry and shape effects cellular behaviour is an exciting research direction? ?, and this study has sought to bridge self-assembly in inanimate anisotropic colloids and hematology. While recent work in the field of inanimate sticky-sphere clusters? ? ? has clearly illuminated self-assembly processes in spherical colloids, our work demonstrates that, while maintaining a level of simplicity, discocyte aggregation introduces aspects of the columnar ordering that gives discotic liquid crystals such interesting properties? . Indeed, we suggest that future research on 
erythrocyte rouleaux will benefit from interactions with the field of lyotropic columnar liquid crystals? ? Similarly, researchers engaged in the synthesis of supercolloidal meso-structures from anisotropic colloids? ? ? ? should look to rouleaux for biomimetic inspiration and consider that red blood cells may make convenient bio-particles for studies of self-assembly. Rouleaux and other hemagglutinates amount to a rich and currently underexplored biophysical system, which navigates the line between interesting entropic soft matter effects and clinical relevance.

\section{Acknowledgments}

HWdH gratefully acknowledges funding from the Natural Sciences and Engineering Research Council (NSERC) in the form of Discovery Grant 2014-06091. We thank Olivera Korculanin for valuable discussions and Lorena Buitrago from the Allen and Frances Adler Laboratory of Blood and Vascular Biology at the Rockefeller University for differential interference contrast microscopy images.

\section{Depletion Pair Potentials Between Two Per- pendicular and Parallel Rouleaux}

In Section ??, we presented the depletant-induced effective pair potentials between two cylinders stacking face-to-face (Eq. ??; Fig ??a) and flanking face-to-side (Eq. ??; Fig ??b). We then used only these two configurations to construct an ensemble of microstates to estimate equilibrium. Furthermore, it was the stability and lifetime of these two states that we investigated in detail in Figs. ?? and ??. We now justify neglecting other configurations.

We continue to limit our considerations to level contacts for which the normals between cylinder faces are either parallel or perpendicular. Any pitch between axes will significantly decrease the overlap volume and, consequently, the pair potential. Likewise, the rouleaux are modelled as smooth and rigid cylinders, while rouleaux may bend and be corrugated as in Fig. ??c-d. Any wrapping or bending may increase the interaction energy.

A.0.0.1 Parallel rouleaux When two cylindrical rouleaux lie parallel to each other (Fig.??a), the depletion pair potential is

$$
\frac{U_{\|}}{k T}=-\frac{3}{2 \pi} \frac{\delta}{R_{d}}\left(\frac{R}{R_{d}}\right)^{2} A\left(\frac{r}{2 R}\right) \phi,
$$

where $\delta(z)=\min \left[\left(L_{1}+L_{2}\right) / 2-|z|, L_{1}, L_{2}\right]$ is the overlap length. In the small-depletant limit, this configuration has a well-depth $U_{\|}^{\text {min }} \approx-(2 \sqrt{2} / \pi)\left(\delta / R_{d}\right)\left(R / R_{d}\right)^{1 / 2} \phi k T$. While $\delta(z)$ allows us to compute the interaction energies when the rouleaux are offset, the potential is deepest when $\delta$ is the shortest rouleau. Letting $m$ be the number of RBCs in the shorter rouleau, $U_{\|}^{\min }=$ $-392.4 m \phi k T$. Comparing to $U_{\mathrm{fs}}^{\mathrm{min}}$ from Section ??, we see that the shorter rouleau must be made of $m>4$ RBCs to be commensurate.

A.0.0.2 Perpendicular rouleaux When two cylindrical rouleaux are in perpendicular contact (Fig.??b), $R_{d} \ll R_{\mathrm{RBC}}$ and (a)
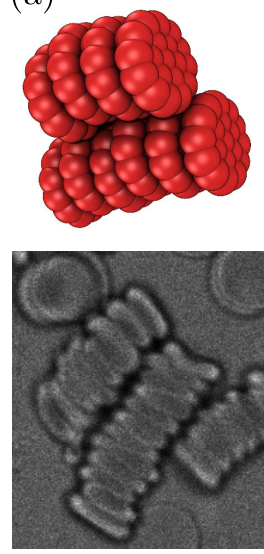

(b)
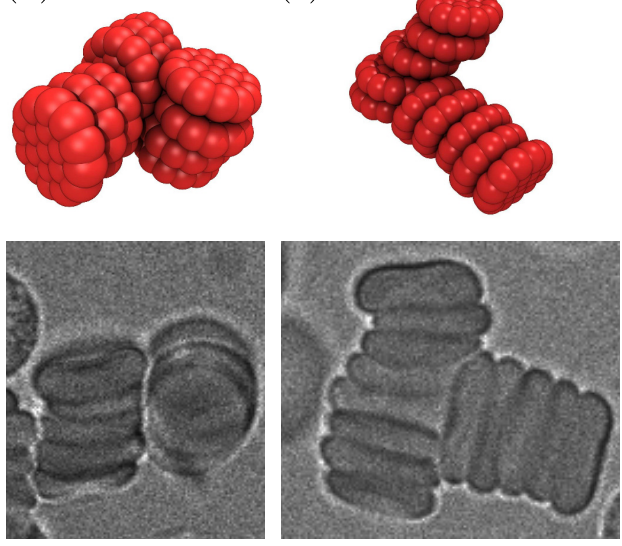

Fig. 9 Typical rouleau-rouleau structures. Above: Simulation snapshots. Below: DIC microscopy images of human erythrocytes. (a) Pair of rouleaux laying side-to-side in parallel with interaction energy $U_{\|}$. (b) Perpendicular rouleaux in bi-secting perpendicular contact with interaction energy $U_{+}$. (c) Two long rouleaux in face-to-side contact, with the same interaction energy $U_{\mathrm{fs}}$ as the quartet in Fig. ??b.

$R_{d} \ll L_{1,2}$, the attractive potential is

$$
\frac{U_{+}}{k T} \approx-\frac{3}{2}\left(\frac{R}{R_{d}}\right)^{3}\left(1+\frac{r}{2 R}\right)\left(1-\frac{r}{2 R}\right)^{2} \phi,
$$

with well-depth $U_{+}^{\min }=-(3 / 2)\left(2 R / R_{d}\right) \phi k T$. This is $-226.5 \phi k T$ in our case, which is approximately twice the sphere-sphere value and nearly nearly an order of magnitude less than the face-to-side value (Fig.??c). If the rouleaux are not pitched with respect to each other but have some tilt, rather than perfect perpendicular or parallel, the well-depth monotonically varies from $U_{+}^{\min }$ to $U_{\|}^{\min }$. 